\author{
Carlotta Pittori · Elisabetta Cavazzuti - Sergio Colafrancesco • Paolo \\ Giommi
}

\title{
Blazar Duty-Cycle at $\gamma$-ray Frequecies: Constraints from Extragalactic Background Radiation and Prospects for AGILE and GLAST
}

Received: date / Accepted: date

\begin{abstract}
We take into account the constraints from the observed extragalactic $\gamma$-ray background to estimate the maximum duty cycle allowed for a selected sample of WMAP Blazars, in order to be detectable by AGILE and GLAST $\gamma$-ray experiments. For the nominal sensitivity values of both instruments, we identify a subset of sources which can in principle be detectable also in a steady state without over-predicting the extragalactic background. This work is based on the results of a recently derived Blazar radio LogN-LogS obtained by combining several multi-frequency surveys.
\end{abstract}

Keywords Blazar · AGN · Extragalactic Background

PACS 95.85.Pw $\cdot 98.54 . \mathrm{Cm} \cdot 98.62 . \mathrm{Ve}$

\section{Introduction}

Blazars are the dominant population of extragalactic sources at microwave, $\gamma$-rays and $\mathrm{TeV}$ energies. They represent $5 \%-8 \%$ of all AGNs and are powerful sources emitting a continuum of electromagnetic radiation from a relativistic jet viewed closely along the line of sight. The large observed variety of Blazar Spectral Energy Distributions (SEDs) can be reproduced, at least in first approximation, by simple Synchrotron Self Compton (SSC) emission model, composed of a synchrotron low-energy component that peaks between the far infrared and the X-ray band, followed by an Inverse Compton component that has its maximum in the hard X-ray band or at higher energies, and may extend into the $\gamma$-ray or

C. Pittori · E. Cavazzuti · P. Giommi

ASI Science Data Center, ASDC c/o ESRIN, via G. Galilei I-00044 Frascati, Italy

Tel.: +39-06-94188878

Fax: +39-06-94188872

E-mail: carlotta.pittori@asdc.asi.it

S. Colafrancesco

INAF-Osservatorio Astronomico di Roma, via di Frascati 33, I-00040 Monteporzio, Italy even the TeV band. Those Blazars where the synchrotron peak is located at low ( infrared) energy are usually called Low energy peaked Blazars or LBL, while those where the synchrotron component reaches the X-ray band are called High energy peaked Blazars or HBL. Blazars may also be subdivided in BL Lacertae types (BL Lacs $20 \%$ of all Blazars), characterized by strong non-thermal emission with no or very weak emission lines and in Flat Spectrum Radio Quasars (FSRQs 80\%) which share the strong non-thermal emission of BL Lacs but also show intense broad line emission. LBL sources, mostly FSRQ and few BL Lacs, are the large majority among Blazars and are usually discovered in radio surveys, while HBL objects all of BL Lac type, are preferentially found in X-ray flux limited surveys.

Despite the relatively low space density of Blazars, their strong emission across the entire electromagnetic spectrum makes them potential candidates as significant contributors to extragalactic Cosmic Backgrounds. Giommi et al. 2005 [1] have recently re-assesed the Blazar contribution to the microwave (CMB), X-ray (CXB), $\gamma$ ray (CGB) and TeV Cosmic backgrounds based on a new estimation of the Blazar radio LogN-LogS, assembled combining several radio and multi-frequency surveys. It was shown that Blazars add a non-thermal component to the overall Cosmic Background that at low frequencies contaminates the CMB fluctuation spectrum. At higher energies $(\mathrm{E}>100 \mathrm{MeV})$ the estimated Blazar collective emission over-predicts the extragalactic background by a large factor, thus implying that Blazars not only dominate the $\gamma$-ray sky (cfr. ref. 2]), but also that their average duty cycle at these frequencies must be rather low.

In this paper we analize a sample of WMAP detected Blazars and we estimate the maximum duty cycle allowed, taking into account the constraints from the observed extragalactic $\gamma$-ray background, in order to be detectable by AGILE and GLAST for the nominal sensitivity values of both instruments. 


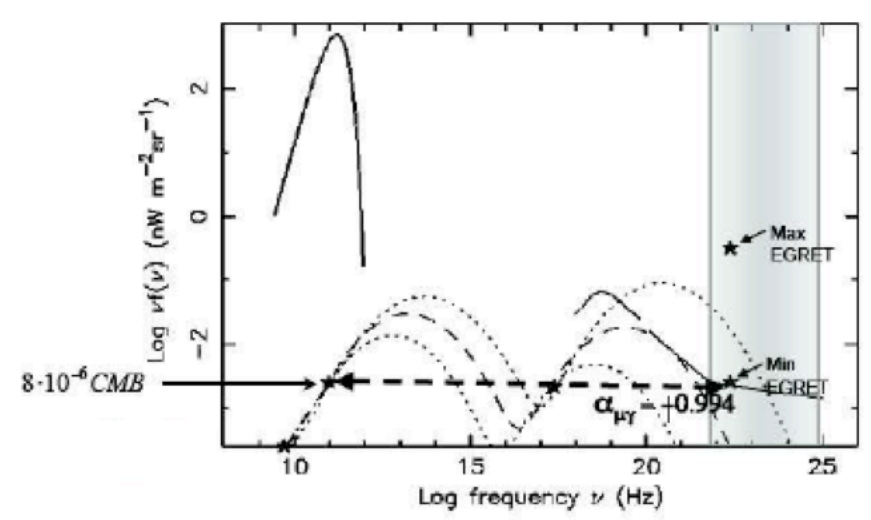

Fig. 1 The possible contribution of LBL Blazars to the Hard $\mathrm{X}$-ray soft $\gamma$-ray Background (shaded area). The three SSC curves corresponds to different $\nu_{\text {peak }}$ values $\left(\log \nu_{\text {peak }}=\right.$ $12.8,13.5$ and 13.8), constrained as described in the text.

\section{Observational Constraints and Blazar $\gamma$-ray Duty Cycle}

The integrated Blazar intensity at microwave frequencies has been computed by using an updated radio $\log \mathrm{N}$ LogS and it has been extrapolated to the hard X-rays and soft $\gamma$-rays by using simple SSC models for the SEDs 1]. Figure 1 shows the CMB, CXB and CGB observed levels, depicted as simple solid lines, together with three SEDs from a simple homogeneous SSC models. The SED parameters are constrained to

- be consistent with the expected integrated flux at 94 $\mathrm{GHz}$,

- have the $\alpha_{\mu x}$ slope equal to the mean value of the WMAP Blazars $\left(\alpha_{\mu x}=1.07\right)$,

- possess a radio spectral slope equal to the average value of the WMAP microwave selected Blazars.

The three curves, forced to pass through the three star symbols graphically representing the three constraints listed above, are characterized by three different synchrotron $\nu_{\text {peak }}$ values.

From Fig. 1 we see that a high value of $\nu_{\text {peak }}$ overpredicts by a large factor the observed hard-X-ray to soft $\gamma$-ray Cosmic Background, whereas a too low value of $\nu_{\text {peak }}$ predicts a negligible contribution. The case Log $\left(\nu_{\text {peak }}\right)=13.5 \mathrm{~Hz}$ predicts $100 \%$ of the Hard-Xray/Soft $\gamma$-ray Cosmic Background. Since the $\log \left(\nu_{\text {peak }}\right)$ values of Blazars in the 1Jy-ARN survey and WMAP catalog peak near 13.5 and range from 12.8 to 13.7 within one sigma from the mean value, the data presently available indicate that Blazars may be responsible for a large fraction, possibly $100 \%$ of the Hard-Xray/Soft $\gamma$-ray Cosmic Background.

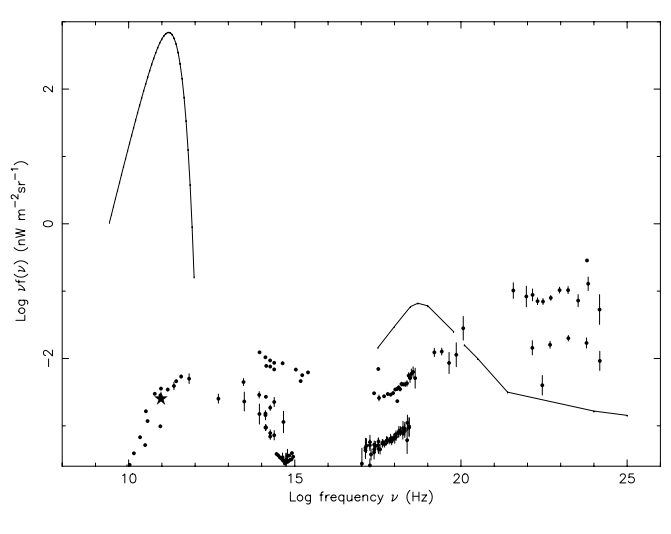

Fig. 2 The CMB, X-ray and $\gamma$-ray cosmic backgrounds with superimposed the SED of the Blazar 3C279 scaled as described in the text.

Blazars are the large majority of the extragalactic $\gamma$-ray $(\mathrm{E}>100 \mathrm{MeV})$ identified sources detected by the EGRET experiment. In order to estimate Blazar contribution to the $\gamma$-ray Cosmic Backgrounds, one can analogously scale the full SED of EGRET detected LBL Blazars, such as that of the well known blazar 3C279, to the integrated Blazar flux intensity at CMB energies. In Fig. 2 we show the SED of 3C279 scaled so that its flux at $94 \mathrm{GHz}$ matches the cumulative emission of the entire Blazar population (star symbol).

From Fig. 2 one can see that while at X-ray frequencies the contribution to the CXB ranges from a few $\%$ to over $10 \%$ in the higher states, the predicted flux at $\gamma$-ray frequencies ranges from about $100 \%$ to several times the observed Cosmic Background intensity. This large excess implies that either 3C279 is highly non representative of the class of Blazars, despite the contribution to the CXB is consistent with other estimates, or its duty cycle at $\gamma$ ray frequencies is very low. The same approach can be followed with other Blazars detected at $\gamma$-ray frequencies. In all EGRET detected WMAP Blazars the SED of LBL Blazars over-predicts the CGB by a large factor.

We define a microwave to $\gamma$-ray slope as

$\alpha_{\mu \gamma}=-\frac{\log \left(f_{94 G H z} / f_{100 M e V}\right)}{\log \left(\nu_{94 G H z} / \nu_{100 M e V}\right)}$,

and a limiting value: $\alpha_{\mu \gamma_{100 \% C G B}}=0.994$ which is the value of an hypotetical source that would produce $100 \%$ of the CGB if representative of the class.

Any source with $\alpha_{\mu \gamma}<0.994$ should have a duty cycle lower than $100 \%$ in order not to overproduce the extragalactic diffuse $\gamma$-ray background.

We estimate the blazar duty cycle by defining

Duty Cycle $=100 \times 10^{-11.41}\left(0.994-\alpha_{\mu \gamma}\right)$,

where $\log \left(\nu_{94 \mathrm{GHz}} / \nu_{100 \mathrm{MeV}}\right)=11.41$.

In the following section we present the preliminary results of our analisis on a sample of WMAP detected 
Blazars and for the nominal sensitivity values AGILE and GLAST instruments we estimate the maximum duty cycle allowed, taking into account the constraints from the observed extragalactic $\gamma$-ray background, in order to be detectable and we identify a subset of sources which can in principle be detectable also in a steady state without over-predicting the background.

\section{Preliminary Results and Discussion}

The subsample we analysed is made of 39 LBL sources, belonging to the 1st year WMAP bright source catalog, selected at high latitude $|b|>30 \mathrm{deg}$ and with measured flux values at $94 \mathrm{GHz}$. The general threshold condition to detect a source flux is: (signal) $\geq n \sigma$, where $\sigma=$ noise, and the signal is equal to $T-B$ (total-background). From error propagation one gets $\sigma=\sqrt{T+B}$, from which is possible to derive a general "handy" sensitivity formula [3] reported below to evaluate AGILE and GLAST sensitivities:

$$
\begin{aligned}
& S\left(E_{i}\right)= \\
& \frac{n^{2}+\sqrt{n^{4}+8 n^{2} F_{\text {diff }} A_{\text {eff }} T 2 \pi\left(1-\cos \theta_{P S F}^{68 \%}\right)}}{2 f A_{\text {eff }} T \Delta E_{i}}
\end{aligned}
$$

Parameters characterising the instruments are the effective area and the PSF $68 \%$ containement radius, that corresponds to the acceptance solid angle value for diffuse background evaluation. The corresponding fraction of accepted signal photons is $\mathrm{f}=0.68$. Note that an $E_{i}$ dependence of these quantities in the formula is to be understood. We set $\mathrm{n}=5$, corresponding to a threshold condition on the signal of $5 \sigma$, we take $\Delta E_{i} \sim E_{i}$ and assume any other efficiency factors $=1$ to give an estimate of limiting sensitivity values for both experiments. Values for AGILE/GRID come from [4] and values for GLAST/LAT come from [5].

We evaluated AGILE sensitivity at high latitude for two exposure times: $\mathrm{T}=10^{6} s \sim 2$ weeks which corresponds to a typical AGILE pointing, and for the nominal lifetime of the mission: $\mathrm{T}=2 \mathrm{yrs}$. GLAST high latitude sensitivity is evaluated for $\mathrm{T}=1 \mathrm{yr}$.

In Table 1 we show our preliminary results for all the 39 sources in the sample. Figs. 3 and 4 show the distribution of the $\alpha_{\mu \gamma}$ and source duty cycle values obtained by using AGILE and GLAST sensitivities.

We note that GLAST in one year would be able to detect all High Latitude WMAP sources in the sample, also in a low-flux steady state with no background constraints. AGILE in two years would be able to detect a few High Latitude WMAP sources with no duty cycle constraints such as 3C279, 3C273 and all other sources in the sample with duty cycle greater than $\sim 20 \%$. AGILE 2 weeks pointing would detect sources in a flaring state with duty cycle in the range $\sim 1-15 \%$.

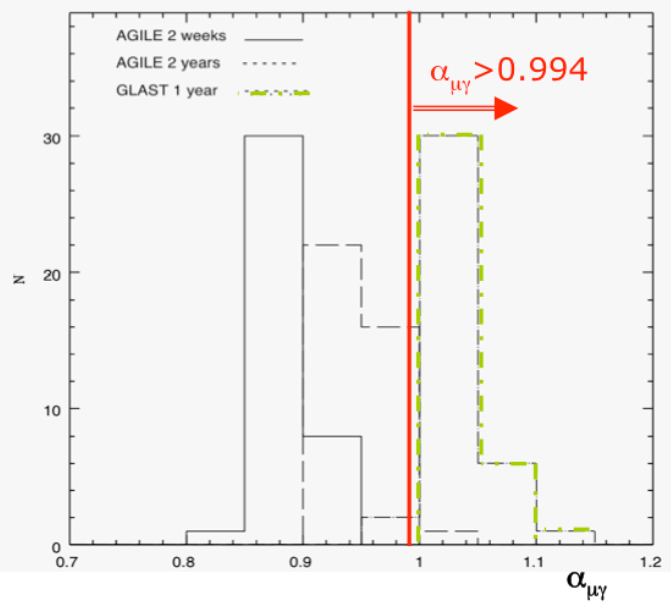

Fig. $3 \alpha_{\mu \gamma}$ histogram for a 2 weeks AGILE pointing, the whole 2 years AGILE sensitivity and the 1 year GLAST sensitivity. $\alpha_{\mu \gamma}>0.994$ corresponds to a duty cycle $>100 \%$ that is no background constraints on the observing capability.

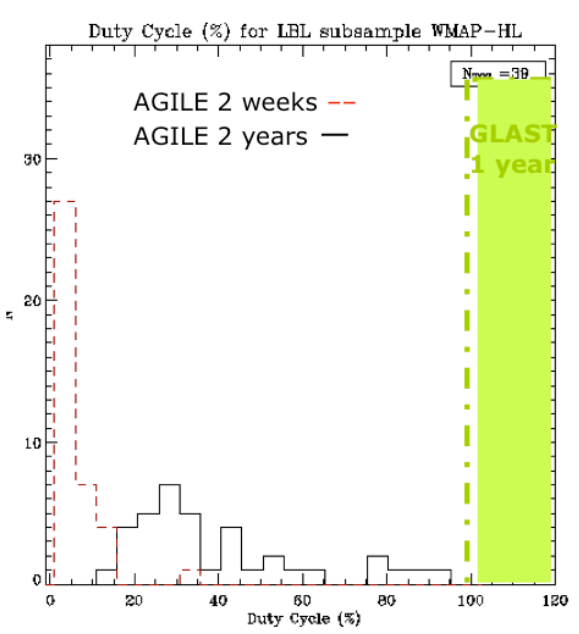

Fig. 4 Duty cycle distribution for the 39 LBL sources of our subsample. GLAST in 1 year will have no background constraints in observing them.

In Tables. 2 and 3 we show the results for the subset of WMAP High Latitude sources which also appear in the third EGRET catalog (3EG). We also compare our results with the GLAST Data Challenge 2 (DC2), corresponding to 55 days of realistic simulated $\gamma$-ray data. We finally show in Fig. 5 the comparison among the Duty Cycle of the High Latitude WMAP subsample with EGRET counterparts. We note that both EGRET and DC2 simulated data correspond to sources with high $\gamma$ ray flux levels, with low duty cycle allowed (in the range $1 \%-15 \%$ ) in order not to overproduce the extragalactic background, assuming that each source is representative of the entire LBL blazar population. Sources in such high 


\begin{tabular}{|c|c|c|c|c|c|c|c|c|c|}
\hline Source Name & $\begin{array}{c}\text { R.A. } \\
(J 2000)\end{array}$ & $\begin{array}{c}\text { Dec } \\
(J 2000)\end{array}$ & $\begin{array}{c}\text { WMAP Flux } \\
94 \mathrm{GHz} \\
(\mathrm{Jy})\end{array}$ & $\begin{array}{c}\alpha_{p r} \\
\text { AGILE } \\
2 \text { weeks }\end{array}$ & $\begin{array}{l}\alpha_{p r} \\
\text { AGILE } \\
2 \text { years }\end{array}$ & $\begin{array}{c}\alpha_{p r} \\
\text { GLAST } \\
1 \text { year }\end{array}$ & $\begin{array}{l}\text { duty cycle } \\
\text { AGILE (\%) } \\
2 \text { w weeks }\end{array}$ & $\begin{array}{c}\text { duty cycle } \\
\text { AGILE (\%) } \\
2 \text { years }\end{array}$ & $\begin{array}{c}\text { duty cycle } \\
\text { GLAST (\%) } \\
1 \text { year }\end{array}$ \\
\hline 1 Jy0208-512 & 021046.2 & -510102 & 1.8 & 0.86 & 0.94 & 1.02 & & 24 & \\
\hline PKS0220-349 & 0222563 & -344127 & 1.1 & 0.84 & 0.92 & 100 & 2 & 15 & 110 \\
\hline PKS 0252-549 & 025329.2 & -544151 & 2.2 & 0.87 & 0.95 & 1.02 & 4 & 29 & 220 \\
\hline PKS 0332-403 & 033413.5 & -400825 & 2.2 & 0.87 & 0.05 & 102 & 4 & 29 & 220 \\
\hline CTA26 & 033930.8 & -014635 & 3.2 & 0.88 & 0.96 & 1.04 & 5 & 43 & 320 \\
\hline PKS 0402-362 & 040353.7 & -360501 & 2.1 & 0.87 & 0.95 & 1.02 & 4 & 28 & 210 \\
\hline PKS 0420-01 & 042315.7 & -012032 & 3.9 & 0.89 & 0.97 & 1.05 & 7 & 52 & 390 \\
\hline $0438-43$ & 044017.1 & -433309 & 2.5 & 0.87 & 0.95 & 1.03 & 4 & 33 & 250 \\
\hline 1 Jy0454-810 & 045005.6 & -810102 & 1.6 & 0.86 & 0.94 & 1.01 & 3 & 21 & 160 \\
\hline 1Jy0454-463 & 045550.7 & -461559 & 3.8 & 0.89 & 0.97 & 1.04 & 6 & 51 & 380 \\
\hline 1Jy0454-234 & 045703.1 & -232451 & 2.7 & 0.88 & 0.96 & 1.03 & 5 & 36 & 270 \\
\hline PKS 0521-365 & 052256.9 & -362754 & 2.3 & 0.87 & 0.95 & 1.03 & 4 & 31 & 230 \\
\hline 1Jy0537-441 & 053851.3 & -440511 & 6.7 & 0.91 & 0.99 & 1.07 & 11 & 89 & 670 \\
\hline $\begin{array}{l}\text { OJ } 287 \\
\text { B2 } 02923+39\end{array}$ & 085448.8 & 200630 & 2.5 & 0.87 & 0.95 & 1.03 & 4 & 33 & 250 \\
\hline B2 $0923+39$ & 092702.9 & 390220 & 3.1 & 0.88 & 0.96 & 1.04 & 5 & 41 & 310 \\
\hline $\begin{array}{l}\text { Tyy } \\
\text { PKS } 105055+015\end{array}$ & $\begin{array}{l}103303.6 \\
105829.5\end{array}$ & $\begin{array}{l}411606 \\
013406\end{array}$ & $\begin{array}{l}2.6 \\
4.6\end{array}$ & $\begin{array}{l}0.87 \\
0.90\end{array}$ & $\begin{array}{l}0.95 \\
0.98\end{array}$ & $\begin{array}{l}1.03 \\
1.05\end{array}$ & $\begin{array}{l}4 \\
8\end{array}$ & $\begin{array}{l}35 \\
61\end{array}$ & $\begin{array}{l}260 \\
460\end{array}$ \\
\hline LB 2136 & 115324.4 & 493109 & 1.3 & 0.85 & 0.93 & 1.00 & 2 & 17 & 130 \\
\hline 4C 29.45 & 115931.7 & 291443 & 2.1 & 0.87 & 0.95 & 1.02 & 4 & 28 & 210 \\
\hline $3 \mathrm{C} 273$ & 122906.3 & 020304 & 9 & 0.92 & 1.00 & 1.08 & 15 & 120 & 901 \\
\hline $3 \mathrm{C} 279$ & 125611.0 & -054719 & 19 & 0.95 & 1.03 & 1.11 & 32 & 253 & 1901 \\
\hline PKS 1335-127 & 133739.7 & -125724 & 3.7 & 0.89 & 0.97 & 1.04 & 6 & 49 & 370 \\
\hline B3 $1417+385$ & 141946.5 & 382147 & 1.5 & 0.85 & 0.93 & 1.01 & 3 & 20 & 150 \\
\hline 1Jy1510-089 & 151250.4 & -090600 & 1.7 & 0.86 & 0.94 & 1.01 & 3 & 23 & 170 \\
\hline 1Jy1606+106 & 160846.0 & 102907 & 3.1 & 0.88 & 0.96 & 1.04 & 5 & 41 & 310 \\
\hline $\begin{array}{l}\text { DA } 406 \\
4 \mathrm{C} 38.41\end{array}$ & $\begin{array}{r}16134340.9 \\
163515.4\end{array}$ & $\begin{array}{l}341246 \\
380804\end{array}$ & $\begin{array}{l}1.4 \\
4.2\end{array}$ & $\begin{array}{l}0.85 \\
0.89\end{array}$ & $\begin{array}{l}0.93 \\
0.97\end{array}$ & $\begin{array}{l}1.01 \\
1.05\end{array}$ & $\begin{array}{l}2 \\
7\end{array}$ & $\begin{array}{l}19 \\
56\end{array}$ & $\begin{array}{l}140 \\
420\end{array}$ \\
\hline 1Jy $1641+399$ & 164258.7 & 394836 & 5.7 & 0.90 & 0.98 & 1.06 & 10 & 76 & 570 \\
\hline $\mathrm{S} 41726+455$ & 172727.5 & 453039 & 1.6 & 0.86 & 0.94 & 1.01 & 3 & 21 & 160 \\
\hline PKS2030-689 & 203548.8 & -684633 & & 0.86 & 0.94 & 1.02 & 3 & 27 & 200 \\
\hline PKS 2121+053 & 212344.4 & 053521 & 2.1 & 0.87 & 0.95 & 1.02 & 4 & 28 & 210 \\
\hline $4 C-02.81$ & 213410.2 & -015316 & 2 & 0.86 & 0.94 & 1.02 & 3 & 27 & 200 \\
\hline 3C 446 & 222547.2 & -045701 & 7.1 & 0.91 & 0.99 & 1.07 & 12 & 95 & 710 \\
\hline CTA102 & 223236.3 & 114350 & 3.1 & 0.88 & 0.96 & 1.04 & 5 & 41 & 310 \\
\hline 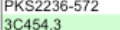 & $\begin{array}{r}2239911.9 \\
225357.6\end{array}$ & $\begin{array}{r}-570101 \\
160852\end{array}$ & $\begin{array}{l}1.4 \\
5.9\end{array}$ & $\begin{array}{l}0.85 \\
0.91\end{array}$ & $\begin{array}{l}0.93 \\
0.98\end{array}$ & $\begin{array}{l}1.01 \\
1.06\end{array}$ & $\begin{array}{r}2 \\
10\end{array}$ & $\begin{array}{l}19 \\
79\end{array}$ & $\begin{array}{l}\begin{array}{l}440 \\
590\end{array} \\
5\end{array}$ \\
\hline 1Jy2254-3 282 & 225805.8 & $\begin{array}{r}160852 \\
-275818\end{array}$ & $\begin{array}{l}5.9 \\
6.3\end{array}$ & 0.91 & 0.99 & $\begin{array}{l}1.00 \\
1.06\end{array}$ & 11 & 84 & $\begin{array}{l}590 \\
630 \\
\end{array}$ \\
\hline 1 Jy $2329-162$ & 233138.6 & -155657 & 1.9 & 0.86 & 0.94 & 1.02 & 3 & 25 & 190 \\
\hline OZ-176 & 2348025 & -163112 & 2.3 & 0.87 & 0.95 & 103 & 4 & 31 & 230 \\
\hline
\end{tabular}

Table 1 Preliminary results for the sample of 39 Low Peaked Blazars with WMAP detection, selected at high latitude and with measured flux values at $94 \mathrm{GHz}$. Green-shadowed sources are those appearing also in 3EG (subsample of 16 sources).

\begin{tabular}{|c|c|c|c|c|c|c|c|}
\hline Source Name & $\begin{array}{c}\text { EGRET } \\
\text { name } \\
\text { 3EG J }\end{array}$ & \begin{tabular}{|c|} 
GLAST \\
DC2 name \\
MRF
\end{tabular} & \begin{tabular}{|c|} 
duty cycle \\
EGRET (\%) \\
F max \\
\end{tabular} & \begin{tabular}{|c|} 
duty cycle \\
EGRET (\%) \\
F min \\
\end{tabular} & $\begin{array}{l}\text { duty cycle } \\
\text { GLAST (\%) } \\
\text { DC2 Fmin }\end{array}$ & $\begin{array}{c}\text { duty cycle } \\
\text { AGILE }(\%) \\
2 \text { years } \\
\end{array}$ & $\begin{array}{c}\text { duty cycle } \\
\text { GLAST }(\%) \\
1 \text { year } \\
\end{array}$ \\
\hline \begin{tabular}{|l|} 
Jy0208-512 \\
\end{tabular} & |0210-5055 & 294 & 1 & 4 & 60 & 24 & 180 \\
\hline CTA26 & |0340-0201 & 282 & 1 & 17 & 107 & 43 & 320 \\
\hline PKS 0420-01 & |0422-0102 & 280 & 4 & 29 & 130 & 52 & 390 \\
\hline 1 Jy $0454-463$ & 0458-4635 & 306 & 11 & 47 & 127 & 51 & 380 \\
\hline 1Jy0454-234 & 0456-2338 & 288 & 13 & 23 & 90 & 36 & 270 \\
\hline 1Jy0537-441 & $0540-4402$ & 310 & 5 & 28 & 223 & 89 & 670 \\
\hline OJ 287 & 0853+1941 & 265 & 11 & 18 & 83 & 33 & 250 \\
\hline 4C 29.45 & $1200+2847$ & 230 & 1 & 19 & 70 & 28 & 210 \\
\hline $3 \mathrm{C} 273$ & $1229+0210$ & 409 & 13 & 73 & 300 & 120 & 901 \\
\hline 3C279 & 1255-0549 & 253 & 5 & 100 & 634 & 253 & 1901 \\
\hline 1Jy1510-089 & 1512-0849 & 220 & 2 & 9 & 57 & 23 & 170 \\
\hline 1Jy1606+106 & $1608+1055$ & 418 & 3 & 10 & 103 & 41 & 310 \\
\hline DA 406 & $1614+3424$ & 260 & 1 & 5 & 47 & 19 & 140 \\
\hline $4 \mathrm{C} 38.41$ & $1635+3813$ & 258 & 3 & 9 & 140 & 56 & 420 \\
\hline CTA102 & $2232+1147$ & 317 & 4 & 18 & 103 & 41 & 310 \\
\hline $3 C 454,3$ & $2254+1601$ & 293 & 3 & 16 & 197 & 79 & 590 \\
\hline
\end{tabular}

Table 2 Results for subsample of sources in 3EG: comparison among max and min observed EGRET fluxes and limiting DC2, AGILE 2-years and GLAST 1-year sensitivities.

\begin{tabular}{|l|c|c|c|c|c|}
\hline Source Name & $\begin{array}{c}\text { EGRET } \\
\text { name } \\
\text { 3EG J }\end{array}$ & $\begin{array}{c}\text { GLAST } \\
\text { DC2 name } \\
\text { MRF }\end{array}$ & $\begin{array}{c}\text { duty cycle } \\
\text { EGRET (\%) } \\
\text { F max }\end{array}$ & $\begin{array}{c}\text { duty cycle } \\
\text { AGILE (\%) } \\
\text { 2 weeks }\end{array}$ & $\begin{array}{c}\text { duty cycle } \\
\text { GLAST (\%) } \\
\text { DC2 }\end{array}$ \\
\hline 1Jy0208-512 & $0210-5055$ & 294 & 1 & 3 & 0 \\
CTA26 & $0340-0201$ & 282 & 1 & 5 & 6 \\
PKS 0420-01 & $0422-0102$ & 280 & 4 & 7 & 3 \\
1Jy0454-463 & $0458-4635$ & 306 & 11 & 6 & 7 \\
1Jy0454-234 & $0456-2338$ & 288 & 13 & 5 & 2 \\
1Jy0537-441 & $0540-4402$ & 310 & 5 & 11 & 3 \\
OJ 287 & $0853+1941$ & 265 & 11 & 4 & 3 \\
4C 29.45 & $1200+2847$ & 230 & 1 & 4 & 6 \\
3C273 & $1229+0210$ & 409 & 13 & 15 & 8 \\
3C279 & $1255-0549$ & 253 & 5 & 32 & 4 \\
1Jy1510-089 & $1512-0849$ & 220 & 2 & 3 & 2 \\
1Jy1606+106 & $1608+1055$ & 418 & 3 & 5 & 1 \\
DA 406 & $1614+3424$ & 260 & 1 & 2 & 0 \\
4C38.41 & $1635+3813$ & 258 & 3 & 7 & 2 \\
CTA102 & $2232+1147$ & 317 & 4 & 5 & 6 \\
3C454.3 & $2254+1601$ & 293 & 3 & 10 & 4 \\
\hline
\end{tabular}

Table 3 Results for subsample of sources in 3EG: comparison among max observed EGRET fluxes, AGILE 2-week sensitivity and simulated GLAST DC2 fluxes.

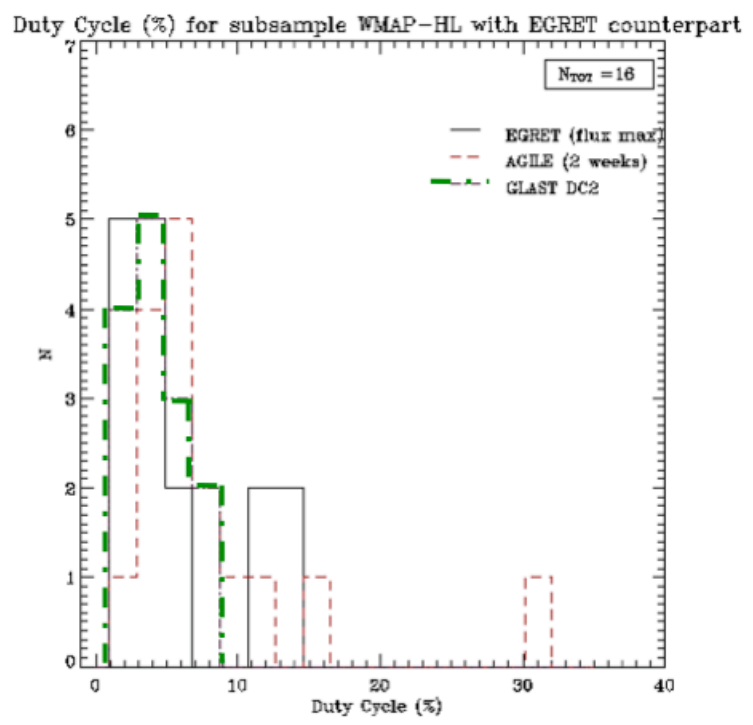

Fig. 5 Duty cycle distribution for the sub-sample of sources with EGRET counterpart: maximum $\gamma$-ray EGRET flux (solid), AGILE sensitivity of one typical pointing (dashed) and GLAST DC2 simulated flux values (dash-dot).

state could also be detected by AGILE in just one pointing ( $\sim 15$ days).

\section{References}

1. Giommi P., Colafrancesco S., Cavazzuti E., Perri M., Pittori C., A\&A 445, 843-855 (2006), and references therein.

2. Padovani P. et al., MNRAS 993, 260, L21.

3. Pittori et al., 2006 in preparation.

4. AGILE Team, http://aqile.rm.iasf.cnr.it

5. GLAST-LAT team, http://wwwgiast.siac.stanford.edu 